\title{
Wenxin Keli attenuates ischemia-induced ventricular arrhythmias in rats: Involvement of L-type calcium and transient outward potassium currents
}

\author{
XI WANG ${ }^{*}$, XIN WANG ${ }^{*}$, YONGWEI GU, TENG WANG and CONGXIN HUANG \\ Department of Cardiology, Renmin Hospital of Wuhan University, Wuhan, P.R. China
}

Received July 20, 2012; Accepted November 8, 2012

DOI: $10.3892 / \mathrm{mmr} .2012 .1195$

\begin{abstract}
Wenxin Keli is the first state-sanctioned traditional Chinese medicine (TCM)-based antiarrhythmic drug. The present study aimed to examine whether long-term treatment with Wenxin Keli reduces ischemia-induced ventricular arrhythmias in rats in vivo, and if so, which mechanisms are involved. Male rats were treated with either saline (control group) or Wenxin Keli for 3 weeks and were subjected to myocardial ischemia for $30 \mathrm{~min}$ with assessment of the resulting ventricular arrhythmias. The L-type calcium current $\left(\mathrm{I}_{\mathrm{Ca}, \mathrm{L}}\right)$ and transient outward potassium current $\left(\mathrm{I}_{\mathrm{to}}\right)$ were measured by the patch clamp technique in normal rat cardiac ventricular myocytes. During the 30-min ischemia, Wenxin Keli significantly reduced the incidence of ventricular fibrillation $(\mathrm{VF})(\mathrm{P}<0.05)$. The number of ventricular tachycardia (VT)+VF episodes and the severity of arrhythmias were significantly reduced by Wenxin Keli administration compared to the control group $(\mathrm{P}<0.05)$. In addition, Wenxin Keli inhibited $\mathrm{I}_{\mathrm{Ca}, \mathrm{L}}$ and $\mathrm{I}_{\text {to }}$ in a concentration-dependent manner. These results suggest that long-term treatment with Wenxin Keli may attenuate ischemia-induced ventricular arrhythmias in rats and that $\mathrm{I}_{\mathrm{Ca}, \mathrm{L}}$ and $\mathrm{I}_{\mathrm{to}}$ may be involved in this attenuation.
\end{abstract}

\section{Introduction}

Ventricular fibrillation (VF) induced by acute myocardial infarction (AMI) frequently occurs without warning, often leading to death within minutes in patients who do not receive prompt medical attention. As is widely known, the

Correspondence to: Professor Congxin Huang, Department of Cardiology, Renmin Hospital of Wuhan University, 238 Jiefang Road, Hubei, Wuhan 430060, P.R. China

E-mail: huangcongxinwhu@163.com

*Contributed equally

Key words: ventricular arrhythmias, L-type calcium current, transient outward potassium current, patch clamp techniques, traditional Chinese medicines cardiac arrhythmia suppression trial (CAST) confirmed that traditional antiarrhythmic drug therapy following myocardial infarction (MI) effectively reduced ventricular premature beats and non-sustained tachycardia, but these drugs were found to increase sudden death and the total mortality rate compared to a placebo (1). Thus, it is critical to develop an effective and safe approach to attenuate ischemia-induced VF in the early phase of AMI in order to reduce sudden cardiac death (SCD). Traditional Chinese medicine (TCM) has documented the use of natural products, primarily plants (the source for over $80 \%$ of the natural products), for over 2,000 years. The substances used medicinally by different ethnic or cultural groups are viewed by researchers as increasingly relevant and important sources of new medicinal products.

Wenxin Keli is the first state-sanctioned TCM-based antiarrhythmic drug and was developed by the Chinese Academy of TCM. Baicalin, the major component of Wenxin Keli, is a flavone, a type of flavonoid, and is found in several species in the genus Scutellaria. This compound has protective effects against heart injury in rats $(2,3)$. Clinical studies have documented the effects of Wenxin Keli in the clinical treatment of arrhythmias, and no significant adverse reactions were observed. Recently, Burashnikov et al (4) found that Wenxin Keli possesses potent anti-atrial fibrillation (AF) properties due to its ability to depress sodium channel-dependent parameters in the atria. However, the effects of Wenxin Keli on ischemia-induced ventricular arrhythmias in vivo remain to be elucidated. In the present study, we demonstrated that long-term oral treatment with Wenxin Keli is capable of attenuating ischemia-induced ventricular arrhythmias in rats, and $\mathrm{I}_{\mathrm{Ca}, \mathrm{L}}$ and $\mathrm{I}_{\text {to }}$ may be involved.

\section{Materials and methods}

Animal preparation and experimental design. All experiments were performed in accordance with the local Institutional Committee on Animal Research of Renmin Hospital of Wuhan University (Wuhan, China) (permit no. 00015816). Rats (250-300 g) were purchased from the Experiment Animal Center of Tongji Medical College, Huazhong University of Science and Technology (Wuhan, China). The investigation complied with the Guide for the Care and Use of Laboratory Animals published by the US National Institutes 
A

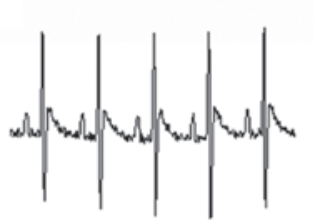

B

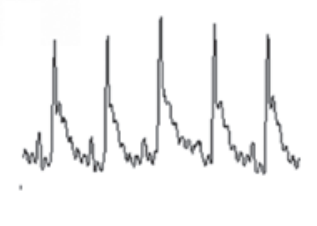

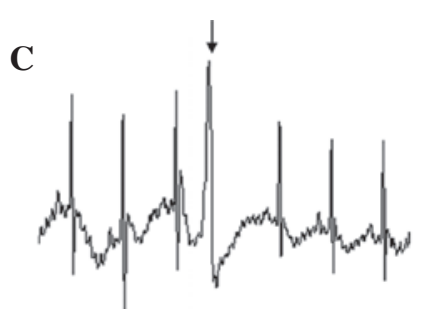

$\mathbf{E}$
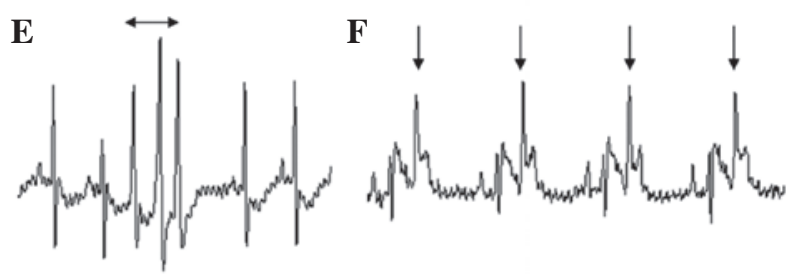

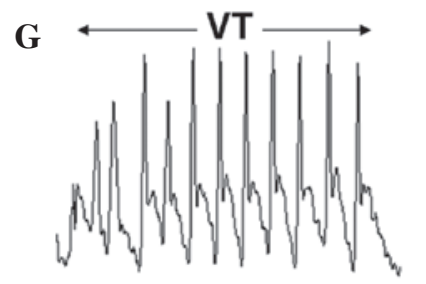

H

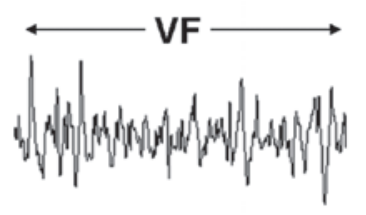

Figure 1. Electrocardiogram recording. (A) At baseline; (B) during coronary artery occlusion; (C) ventricular ectopic beat (VEB); (D) couplet; (E) triplet; (F) bigeminy; $(\mathrm{G})$ ventricular tachycardia (VT); $(\mathrm{H})$ ventricular fibrillation (VF).

of Health (NIH publication no. 85-23, revised 1996). Room temperature was maintained at $23^{\circ} \mathrm{C}$ with constant humidity (55\%), and the lights were maintained on a 12-h light/dark cycle (8:00 am-8:00 pm light/8:00 pm-8:00 am dark). A total of 34 rats were randomly divided into three groups: Group 1, sham-operated group $(n=8)$, rats underwent surgical procedures without coronary artery ligation; Group 2, control group $(n=13)$, saline was administered for 3 weeks by gavage prior to coronary artery occlusion; Group 3, Wenxin Keli group $(\mathrm{n}=13)$, Wenxin Keli (8 g/kg, qd, gavage) was administered for 3 weeks prior to coronary artery occlusion.

After being anesthetized with sodium pentobarbital [40 mg/kg, intraperitoneally (IP)], the rats were ventilated artificially via a tracheal cannula using a constant volume rodent ventilator (tidal volume, $3.0 \mathrm{ml}$; respiratory rate, 70 strokes $/ \mathrm{min}$ ). The right common carotid artery was cannulated to measure the mean arterial blood pressure (MBP). Lead II of the electrocardiogram was monitored with subcutaneous stainless steel electrodes. A computer-based EP system (LEAD2000B; Jinjiang Ltd., Chengdu, China) was used to record the heart rate and the electrocardiogram. Under sterile conditions, a left thoracotomy was performed in the fourth intercostal space. After pericardiotomy, a 5-0 prolene suture was tied around the left anterior descending coronary artery at $2-3 \mathrm{~mm}$ from its origin. A successful myocardial ischemia model was confirmed by ST segment elevation in Lead II and by regional cyanosis of the myocardial surface.

Assessment of ventricular arrhythmias. Ischemia-induced ventricular arrhythmias were identified in accordance with the Lambeth Conventions as in our previous study (5) (Fig. 1). Ventricular ectopic beats (VEBs) were defined as identifiable premature QRS complexes. Ventricular tachycardia (VT) was defined as the occurrence of $\geq 4$ consecutive VEBs at a rate faster than the resting sinus rate. VF was defined as unidentifiable and low voltage QRS complexes. Other multipart forms of VEBs, such as bigeminy, couplets (two consecutive VEBs) and triplets (three consecutive VEBs), were evaluated as separate episodes (Fig. 1). VF may be sustained or may spontaneously revert to a normal sinus rhythm. VF lasting for $>5$ min was considered irreversible.

The severity of the arrhythmias was quantified by the following scoring system (6,7): a total of 0-50 VEBs with no other arrhythmias during the 30-min ischemia period resulted in a score of 0 ; a total of 50-500 VEBs in a score of 1; a total of $>500 \mathrm{VEBs}$ or one episode of spontaneously reversible VT or VF in a score of 2; a total of 2-30 episodes of spontaneously reversible VT and/or VF in a score of 3; a total of $>30$ episodes of spontaneously reversible VT and/or VF in a score of 4; and irreversible $\mathrm{VF}$ in a score of 5.

\section{Whole-cell patch clamp recording}

Isolation of cardiac ventricular myocytes and patch clamp recordings. Ventricular myocytes were isolated by collagenase type 2 (Type II; Sigma, St. Louis, MO, USA) perfusion from normal adult rats as previously described (8). All steps were performed at $37^{\circ} \mathrm{C}$ in solutions gassed with $95 \% \mathrm{O}_{2}+5 \% \mathrm{CO}_{2}$. The ventricles were cut off, cut into small pieces and gently stirred in Tyrode's solution plus $1 \mathrm{mg} / \mathrm{ml}$ bovine serum albumin to collect ventricular myocytes.

Membrane currents were obtained and analyzed with an EPC-9 patch clamp amplifier (HEKA Electronik, Lambrecht, Germany) in the whole-cell mode by the Pulse/Pulsefit software program (HEKA Elektronik). Single cardiac ventricular myocytes were placed in the experimental chamber $(1.5 \mathrm{ml})$ mounted on the stage of an inverted microscope (IX70; Olympus, Tokyo, Japan) and perfused with external solution including different concentrations of Wenxin Keli (1 and $10 \mathrm{~g} / \mathrm{l})$ 
A

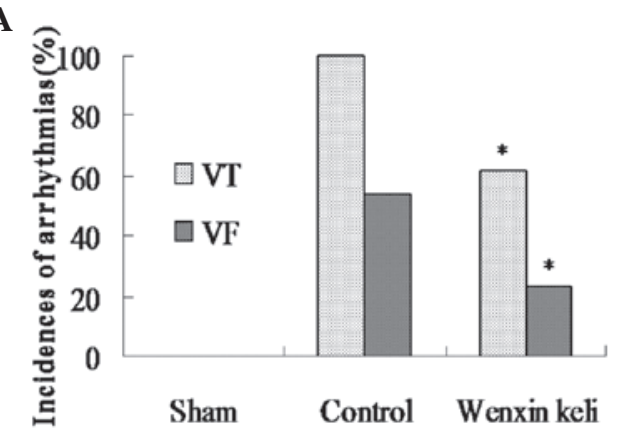

C

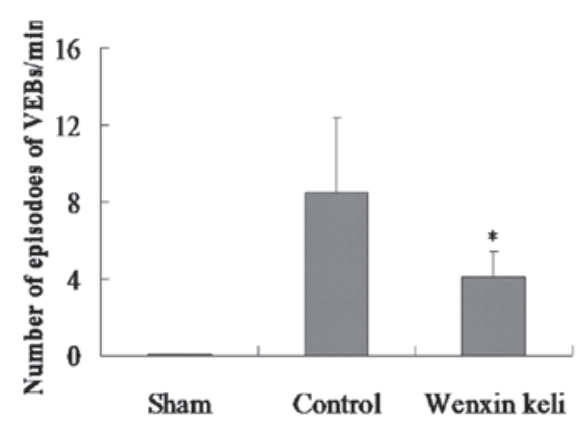

B

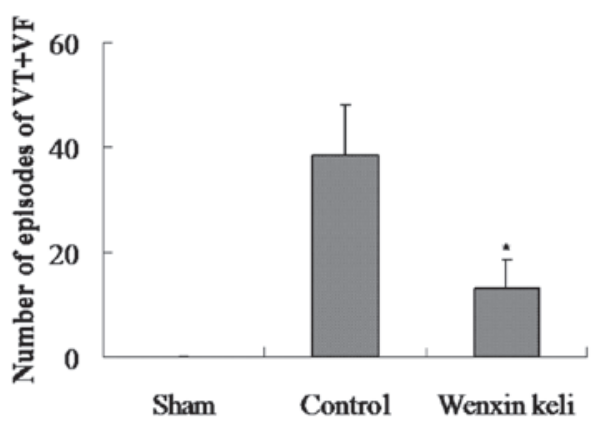

D

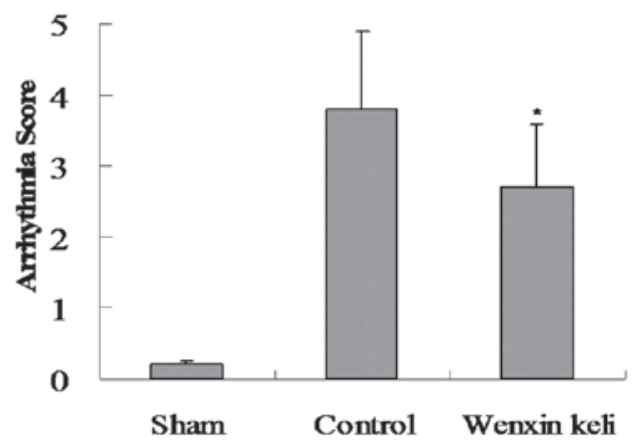

Figure 2. Effects of Wenxin Keli on ischemia-induced ventricular arrhythmias and the distribution of the arrhythmia score during 30-min ischemia in the sham, control and Wenxin Keli groups. (A) Incidence of VT and VF. (B) Number of episodes of VT+VF. (C) Number of episodes of VEBs/min. (D) Distribution of the arrhythmia score. ${ }^{*} \mathrm{P}<0.05$ vs. the control group. VT, ventricular tachycardia; VF, ventricular fibrillation; VEB, ventricular ectopic beats.

for $5 \mathrm{~min}$ at a rate of $2-3 \mathrm{ml} / \mathrm{min}$. The measurements were performed at room temperature $\left(20-25^{\circ} \mathrm{C}\right)$. Glass microelectrodes were made using two-stage pulling with a resistance of 3.0-5.0 M $\Omega$ on microelectrodes (PB-7; Narishige, Tokyo, Japan) filled with internal solution. The mean capacitance of the cells was $92.92 \pm 35.52 \mathrm{pF}$, and the series resistances were $<25 \mathrm{M} \Omega$. All currents were digitally sampled at $10 \mathrm{kHz}$, low-pass filtered at $1 \mathrm{kHz}$, and saved on a hard drive for post hoc analysis.

Measurement of $I_{C a, L}$ and $I_{t o}$. $\mathrm{I}_{\mathrm{Ca}, \mathrm{L}}$ was recorded using a whole-cell patch clamp configuration. The pipette solution contained $120 \mathrm{mM} \mathrm{CsCl}, 1.0 \mathrm{mM} \mathrm{CaCl}_{2}, 5.0 \mathrm{mM} \mathrm{MgCl}$, $5.0 \mathrm{mM} \mathrm{Na}{ }_{2}$ ATP, $11 \mathrm{mM}$ EGTA, $10 \mathrm{mM}$ HEPES and $11 \mathrm{mM}$ glucose, adjusted to $\mathrm{pH} 7.2$ with $\mathrm{CsOH}$. The external solution was Tyrode's solution $(135 \mathrm{mM} \mathrm{NaCl}, 5.4 \mathrm{mM} \mathrm{KCl}$, $1.8 \mathrm{mM} \mathrm{CaCl}_{2}, 1.0 \mathrm{mM} \mathrm{MgCl} 2,0.33 \mathrm{mM} \mathrm{NaH} \mathrm{PO}_{4}, 10 \mathrm{mM}$ HEPES and $10 \mathrm{mM}$ glucose, adjusted to $\mathrm{pH} 7.35$ with $\mathrm{NaOH}$ ), including 1 or $10 \mathrm{~g} / 1$ Wenxin Keli. In order to estimate the spontaneous decline of $\mathrm{I}_{\mathrm{Ca}, \mathrm{L}}$ with time (run-down) during the first $5 \mathrm{~min}$ of recording, we added $5 \mathrm{mmol} / \mathrm{l} \mathrm{MgATP}$ to the pipette solution and commenced data acquisition after 5-15 min of equilibration between the pipette solution and the intracellular contents.

The external solution used to record $\mathrm{I}_{\mathrm{to}}$ contained $30 \mathrm{mM}$ $\mathrm{NaCl}, 110 \mathrm{mM}$ choline chloride, $5.4 \mathrm{mM} \mathrm{KCl}, 1.0 \mathrm{mM} \mathrm{MgCl}$, $0.33 \mathrm{mM} \mathrm{NaH}_{2} \mathrm{PO}_{4}, 10 \mathrm{mM}$ HEPES, $10 \mathrm{mM}$ glucose and $0.3 \mathrm{mM} \mathrm{CdCl}$, adjusted to $\mathrm{pH} 7.35$ with $\mathrm{NaOH}$. The pipette solution used to record $\mathrm{I}_{\text {to }}$ contained $45 \mathrm{mM} \mathrm{KCl}, 85 \mathrm{mM}$ K-aspartate, $5 \mathrm{mM}$ Na-pyruvate, $5.0 \mathrm{mM} \mathrm{MgATP,} 10 \mathrm{mM}$ EGTA, $10 \mathrm{mM}$ HEPES and $11 \mathrm{mM}$ glucose, adjusted to pH 7.2 with $\mathrm{KOH}$. HEPES, $\mathrm{Na}_{2} \mathrm{ATP}, \mathrm{CsCl}$, EGTA and $\mathrm{CsOH}$ were purchased from Sigma. All the other chemicals were of analytical grade. Wenxin Keli was provided by the Shandong Buchang Pharmaceutical Company Co., Ltd. (Beijing, China).
Data analysis. All values were presented as the means \pm SD. The incidence of VT and VF was compared using the Fisher's exact test, and the arrhythmia scores were analyzed with the Kruskal-Wallis test. Patch clamp data were analyzed using one-way analysis of variance (ANOVA). Statistical significance was defined as $\mathrm{P}<0.05$.

\section{Results}

Ventricular arrhythmias during ischemia. The MBP and heart rate (HR) were continuously recorded during the experiments, and the average MBP and heart rate during the 30-min baseline and 30-min ischemia periods were calculated. No significant differences were found in the HR and MBP between the groups ( $\mathrm{P}>0.05)$ at baseline. The MBP and heart rate after 30-min ligation were lower than those before 30-min ligation, but the differences were not statistically significant $(P>0.05)$. In this model of ischemia, severe ventricular arrhythmias peaked at 0-30 min following coronary artery ligation. Fig. 1 shows the different ventricular arrhythmias during the 30-min ischemia.

Incidence of VT and VF and number of episodes of VT+VF. In the myocardial ischemia group, VT was observed in $100 \%$ $(13 / 13)$ of the rat hearts, and $53.84 \%(7 / 13)$ of the hearts exhibited VF. The administration of Wenxin Keli attenuated the incidence of VT to $61.54 \%(8 / 13)$ and that of VF to $23.08 \%$ (3/13) compared with the control group (Fig. 2A). The number of episodes of $\mathrm{VT}+\mathrm{VF}$ in the Wenxin Keli group (13.2 \pm 5.3$)$ was significantly lower compared to that in the control group

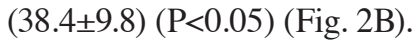

Number of episodes of VEBs/min and severity of arrhythmias. The number of episodes of VEBs/min in the Wenxin Keli 
A Control

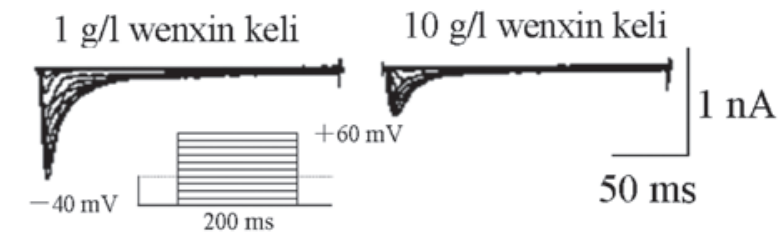

$10 \mathrm{~g} / \mathrm{l}$ wenxin keli

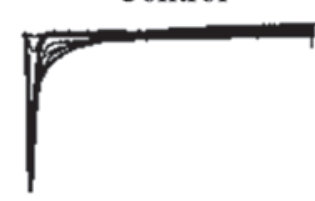

B

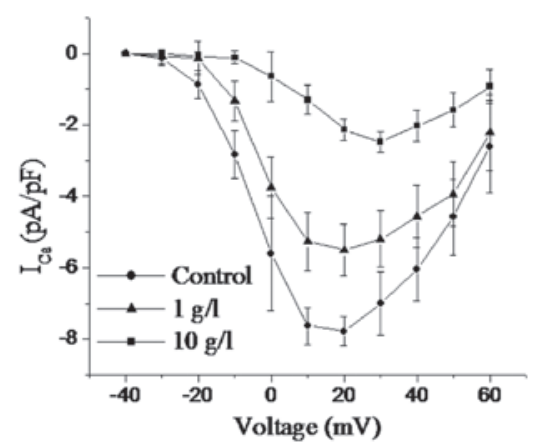

D

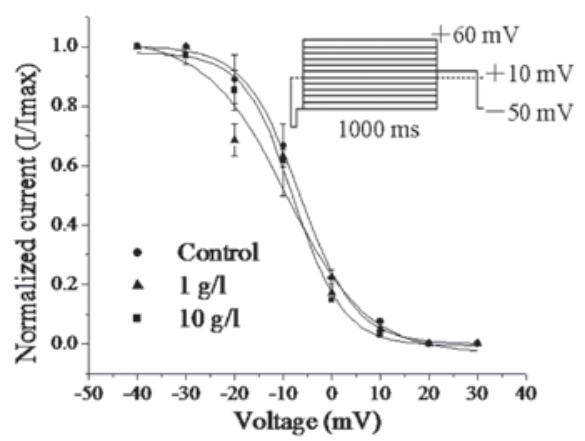

C

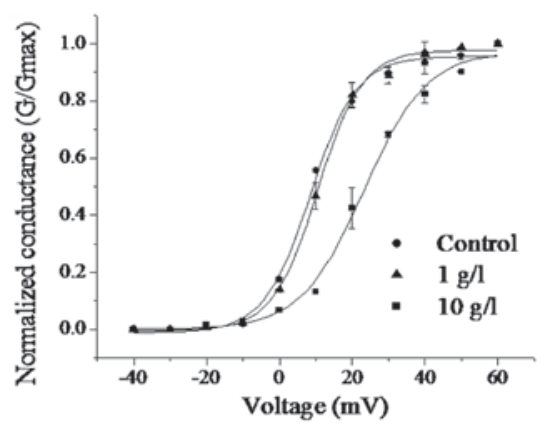

E

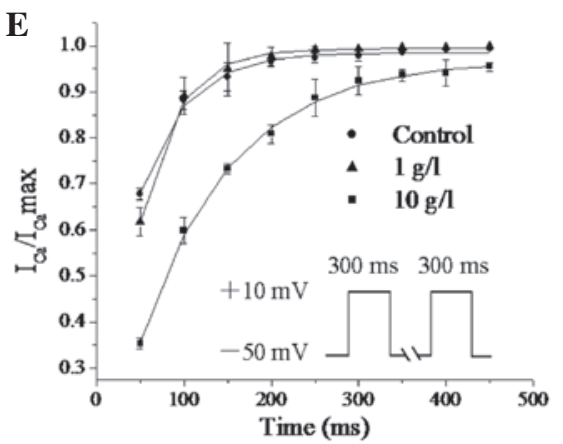

Figure 3. Effect of Wenxin Keli on $\mathrm{I}_{\mathrm{Ca}, \mathrm{L}}$. (A) Original recordings of $\mathrm{I}_{\mathrm{Ca}, \mathrm{L}}$ for the control, 1 and $10 \mathrm{~g} / 1$ Wenxin Keli groups. (B) Current-voltage (I-V) correlation for $\mathrm{I}_{\mathrm{Ca}, \mathrm{L}}$ in rat ventricular myocytes. (C) Mean values for the voltage dependence of the activation $(\mathrm{G} / \mathrm{Gmax})$ of $\mathrm{I}_{\mathrm{Ca}, \mathrm{L}}$. Curves were fitted to the Boltzmann distribution. (D) Mean values for the voltage dependence of the inactivation (I/Imax) of $\mathrm{I}_{\mathrm{Ca}, \mathrm{L}}$. Curves were fitted to the Boltzmann distribution. (E) Mean value curves for the recovery of $\mathrm{I}_{\mathrm{Ca}, \mathrm{L}}$ following inactivation. Recovery curves were fitted to a mono-exponential function.

group $(4.1 \pm 1.3)$ was significantly decreased compared to that in the control group $(8.5 \pm 3.9)(\mathrm{P}<0.05)(\mathrm{Fig} .2 \mathrm{C})$. The severity of ventricular arrhythmias was significantly attenuated by Wenxin Keli $(2.7 \pm 0.9)$ compared to the severity in the myocardial ischemia group $(3.8 \pm 1.1)(\mathrm{P}<0.05)$ (Fig. 2D).

Wenxin Keli inhibits $I_{C a, L}$. Fig. 3A shows the voltage-dependent $\mathrm{I}_{\mathrm{C}, \mathrm{L}}$ traces recorded in the absence and presence of Wenxin Keli (protocol, HP=-40, $200 \mathrm{~ms}$ pulses of voltages between -40 and $+60 \mathrm{mV}$ in $10 \mathrm{mV}$ steps preceded by a $50 \mathrm{~ms}$ prepulse of $-40 \mathrm{mV}$ ). Wenxin Keli markedly reduced the amplitude of $\mathrm{I}_{\mathrm{Ca}, \mathrm{L}}$. The current-voltage (I-V) correlations for the $\mathrm{I}_{\mathrm{Ca}, \mathrm{L}}$ density shown in Fig. 3B indicate that Wenxin Keli significantly inhibited $\mathrm{I}_{\mathrm{Ca}, \mathrm{L}}$ at -10 to $+60 \mathrm{mV}$ in a concentration-dependent manner.

The activation conductance variable $(\mathrm{G} / \mathrm{Gmax})$ of $\mathrm{I}_{\mathrm{Ca}, \mathrm{L}}$ was determined from the I-V relationship for each cell (Fig. 3B) and was fitted to the Boltzmann distribution to obtain the half activation (V0.5) and slope values. The V0.5 of $\mathrm{I}_{\mathrm{Ca}, \mathrm{L}}$ activation positively shifted by $9.6 \mathrm{mV}$ in the cells treated with $10 \mathrm{~g} / \mathrm{l}$ of Wenxin Keli $(12.78 \pm 8.7 \mathrm{mV}$ in the control group to $22.38 \pm 5.1 \mathrm{mV}$ in the Wenxin Keli group; $\mathrm{n}=5 ; \mathrm{P}<0.05$ ) (Fig. 3C), whereas no change was observed when using $1 \mathrm{~g} / \mathrm{l}$ of Wenxin Keli. The values of the variables (I/Imax) for the voltage-dependent inactivation of $\mathrm{I}_{\mathrm{Ca}, \mathrm{L}}$ were determined with the double-pulse protocol (a 1,000 ms prepulse of potentials between -50 and $+60 \mathrm{mV}$ in $10 \mathrm{mV}$ steps, followed by a fixed $400 \mathrm{~ms}$ test pulse of $10 \mathrm{mV}$ ) (Fig. 3D), and these data were also fitted to the Boltzmann distribution. The V0.5 of $\mathrm{I}_{\mathrm{Ca}, \mathrm{L}}$ inactivation was not significantly changed by the administration of Wenxin Keli.

The time-dependent recovery of $\mathrm{I}_{\mathrm{Ca}, \mathrm{L}}$ following inactivation was studied with the double-pulse protocol consisting of two identical pulses (holding potential from -50 to $+10 \mathrm{mV}$ for $300 \mathrm{~ms}$ ) in variable intervals from 50 to $500 \mathrm{~ms}$ in $50 \mathrm{~ms}$ increments (Fig. 3E). The recovery curves were fitted to a mono-exponential function. The recovery time constant of $\mathrm{I}_{\mathrm{Ca}, \mathrm{L}}$ was slowed by $10 \mathrm{~g} / \mathrm{l}$ Wenxin Keli $(55.76 \pm 5.98 \mathrm{~ms}$ in control, $104.13 \pm 4.71 \mathrm{~ms}$ in $10 \mathrm{~g} / \mathrm{l} ; \mathrm{n}=6 ; \mathrm{P}<0.05)$, whereas no change was observed for $1 \mathrm{~g} / \mathrm{l}$ Wenxin Keli $(59.82 \pm 7.24 \mathrm{~ms})$. These results demonstrate that Wenxin Keli inhibits $\mathrm{I}_{\mathrm{Ca}, \mathrm{L}}$ by decelerating the activation process and delaying recovery from inactivation without changing the inactivation process.

Wenxin Keli inhibits $I_{t o}$. Fig. 4A illustrates the voltage-dependent $\mathrm{I}_{\mathrm{to}}$ trace in the absence and presence of Wenxin Keli $(500 \mathrm{~ms}$ depolarization step pulses from -40 to $+60 \mathrm{mV}$ with a step size of $10 \mathrm{mV}$ ). Wenxin Keli at $10 \mathrm{~g} / 1$ markedly reduced the amplitude of $\mathrm{I}_{\mathrm{to}}$. The I-V relationship for the $\mathrm{I}_{\mathrm{to}}$ density, shown in Fig. 4B, indicated that Wenxin Keli inhibited $\mathrm{I}_{\mathrm{to}}$ in a concen- 


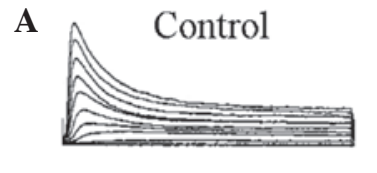

B

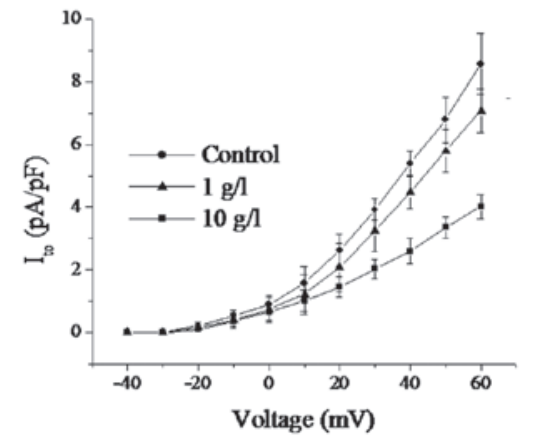

D

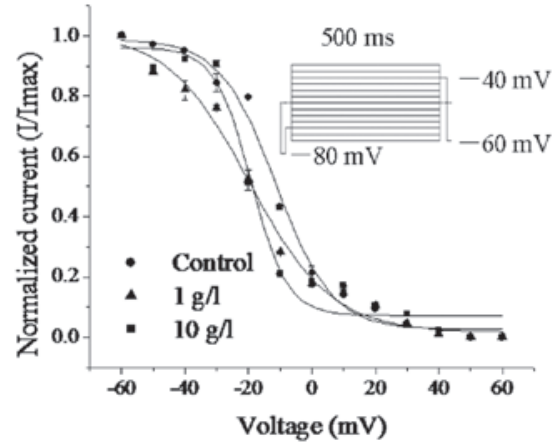

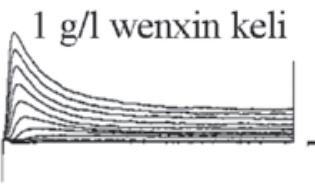

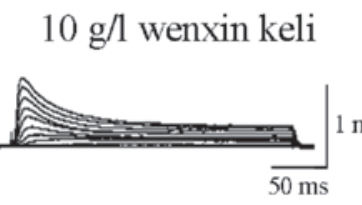

C

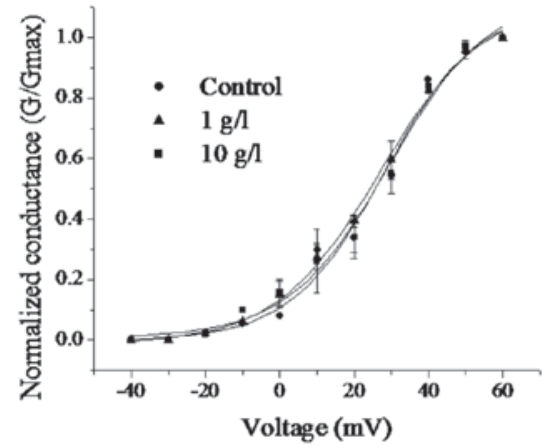

$\mathbf{E}$

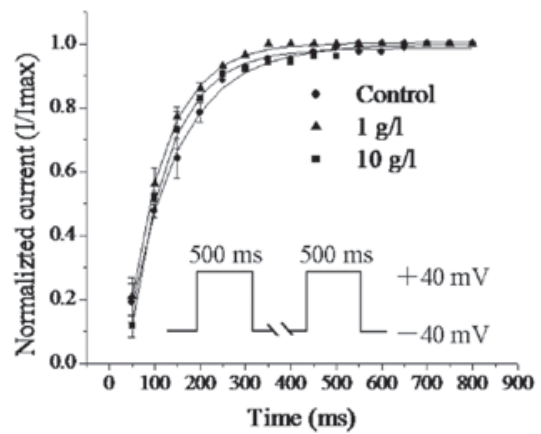

Figure 4. Effect of Wenxin Keli on $\mathrm{I}_{\mathrm{to}}$. (A) Original recordings of $\mathrm{I}_{\mathrm{to}}$ for the control, 1 and $10 \mathrm{~g} / 1$ Wenxin Keli groups. (B) $\mathrm{I}-\mathrm{V}$ relationship for $\mathrm{I}_{\mathrm{to}}$ in rat ventricular myocytes. (C) Mean values for the voltage dependence of the activation (G/Gmax) of $\mathrm{I}_{\mathrm{to}}$. Curves were fitted to the Boltzmann distribution. (D) Mean values for the voltage dependence of the inactivation (I/Imax) of $\mathrm{I}_{\mathrm{t} 0}$. Curves were fitted to the Boltzmann distribution. (E) Mean value curves for the recovery of $\mathrm{I}_{\mathrm{to}}$ following inactivation. Recovery curves were fitted to a mono-exponential function.

tration-dependent manner. The peak amplitude decreased to $15.31 \pm 7.21 \%$ at $1 \mathrm{~g} / \mathrm{l}$ and to $53.25 \pm 4.74 \%$ at $10 \mathrm{~g} / \mathrm{l}(\mathrm{n}=6, \mathrm{P}<0.05)$.

By fitting the activation process of $\mathrm{I}_{\text {to }}$ to the Boltzmann distribution, we found that Wenxin Keli (1 and $10 \mathrm{~g} / \mathrm{l})$ had no significant effect on voltage-dependent activation (Fig. 4C). Steady-state inactivation was analyzed using a double-pulse protocol: a 1,000 ms prepulse of potentials between -60 and $+60 \mathrm{mV}$ in $10 \mathrm{mV}$ steps, followed by a fixed $400 \mathrm{~ms}$ test pulse of $+40 \mathrm{mV}$. The V0.5 of voltage-dependent inactivation was negatively shifted by treatment with Wenxin Keli $(-11.30 \pm 2.6 \mathrm{mV}$ for control, $-19.75 \pm 3.02 \mathrm{mV}$ for $1 \mathrm{~g} / \mathrm{l}$, and $-19.21 \pm 4.15 \mathrm{mV}$ for $10 \mathrm{~g} / \mathrm{l}, \mathrm{n}=6, \mathrm{P}<0.05$ ) (Fig. 4D).

Recovery of $\mathrm{I}_{\text {to }}$ after inactivation was investigated with a paired-pulse protocol $(\mathrm{HP}=-80 \mathrm{mV}$, a $500-\mathrm{ms}$ conditioning pulse of $+40 \mathrm{mV}$ was separated from a 50 -ms test pulse of $-40 \mathrm{mV}$ by a gradually prolonged recovery interval between 50 and $800 \mathrm{~ms}$ ) (Fig. 4E), and the recovery curves were fitted with a mono-exponential function. No significant changes in the recovery time constants were observed. These results demonstrate that Wenxin Keli inhibits $I_{t o}$ by accelerating its inactivation without changing its activation process or recovery from inactivation.

\section{Discussion}

Ventricular arrhythmias, particularly spontaneous or induced ventricular tachyarrhythmias and fibrillation, are frequently observed post-infarction in various animal models of MI $(9,10)$. The majority of sudden cardiac deaths are thought to be due to ventricular arrhythmias. Thus, the treatment of ventricular arrhythmias, particularly VF, is important in order to reduce the risk of sudden cardiac death post-infarction. In the present study, Wenxin Keli was shown to prevent ventricular arrhythmias in vivo following long-term administration in a rat model of MI. Our data also demonstrated that the antiarrhythmic effect of Wenxin Keli is associated with the inhibition of $\mathrm{I}_{\mathrm{Ca}, \mathrm{L}}$ and $\mathrm{I}_{\mathrm{to}}$.

Traditional antiarrhythmic drugs may paradoxically precipitate lethal arrhythmias; these drugs occasionally intensify rather than inhibit arrhythmias $(11,12)$. Basic research into the clinical application of traditional Chinese medicine has been conducted. It has been recognized worldwide that traditional Chinese medicine has broad clinical prospects due to its advantages with respect to multiple targets, significant efficacy and safety. Wenxin Keli is useful for treating functional arrhythmia and arrhythmia as a complication of infective cardiomyopathy in the elderly and children. It has been shown that the combined use of Wenxin Keli and amiodarone has a better effect on the conversion rate of $\mathrm{AF}$, shortening the conversion time and decreasing the required dosage of amiodarone in treating AF, compared with treatment with amiodarone alone. The use of Wenxin Keli also protects against the adverse effects of the long-term use of amiodarone $(13,14)$. In addition, Wenxin Keli is capable of greatly improving isoproterenol-induced cardiac dysfunc- 
tion and protecting against aconitine-induced arrhythmia in rats (15). Moreover, Wenxin Keli produces atrial-selective depression of $\mathrm{I}_{\mathrm{Na}}$-dependent parameters in isolated canine coronary perfused preparations and effectively suppresses AF and prevents its induction $(4,16)$. Therefore, it is implied that Wenxin Keli has good clinical prospects.

It is generally accepted that cardiac repolarization and refractoriness are determined by the balance of inward $\mathrm{Ca}^{2+}$ currents and outward $\mathrm{K}^{+}$currents. The L-type $\mathrm{Ca}^{2+}$ channel is considered to be the primary route for calcium influx into cardiac myocytes and an important determinant of calcium homeostasis. The increased $\mathrm{I}_{\mathrm{Ca}, \mathrm{L}}$ may contribute to the prolongation of the action potential duration and increase the frequency of early afterdepolarizations (EADs), as demonstrated for L-type $\mathrm{Ca}^{2+}$ channel agonists (17-21). Pathological remodeling of the myocardium depends on the persistent activation of L-type calcium channels, which alters calcium homeostasis and is responsible for the induction of hypertrophic growth $(22,23)$. In addition, more calcium entered the cell through the L-type $\mathrm{Ca}^{2+}$ channel during depolarization, leading to calcium overload and triggering cell death signals (24). $I_{\text {to }}$ is a key regulator of phase one action potential repolarization and is the primary cause of spike-and-dome morphology (25). In addition, $\mathrm{I}_{\mathrm{to}}$ is important in human ventricle repolarization. Its voltage-dependent activation and inactivation kinetics are much faster than those of other cardiac K currents. Increased $\mathrm{I}_{\text {to }}$ density may eliminate the plateau, which is the primary mechanism responsible for the occurrence and maintenance of VF. The data from our study demonstrate that Wenxin Keli significantly inhibits $\mathrm{I}_{\mathrm{Ca}, \mathrm{L}}$ and $\mathrm{I}_{\mathrm{to}}$ in adult rat ventricular myocytes. This substance reduced the amplitude of $\mathrm{I}_{\mathrm{Ca}, \mathrm{L}}$, decelerated the activation process and slowed down its recovery from inactivation, whereas the inactivation process remained unaffected. In addition, Wenxin Keli inhibited $\mathrm{I}_{\text {to }}$ and accelerated its inactivation without changing the activation process or the recovery of its inactivation. These effects of Wenxin Keli on $\mathrm{I}_{\mathrm{Ca}, \mathrm{L}}$ and $\mathrm{I}_{\mathrm{to}}$ may be protective against cardiac fibrillation.

In conclusion, the present study demonstrates that Wenxin Keli attenuated ischemia-induced ventricular arrhythmias and inhibited $\mathrm{I}_{\mathrm{Ca}, \mathrm{L}}$ and $\mathrm{I}_{\mathrm{to}}$. The regulation of $\mathrm{I}_{\mathrm{Ca}, \mathrm{L}}$ and $\mathrm{I}_{\mathrm{to}}$ contributed, at least in part, to the antiarrhythmic action of Wenxin Keli.

\section{Acknowledgements}

This study was financially supported by the Fundamental Research Funds for the Central Universities (no. 201030201 01000195 and 201130202020003). The authors are grateful to Shandong Buchang Pharmaceutical Co., Ltd. for generously providing Wenxin Keli. The authors would also like to thank Professor Na Luo (School of Foreign Language, Wuhan University of Science and Technology, Wuhan, China) for providing assistance in writing the manuscript.

\section{References}

1. The cardiac arrhythmia suppression trial. N Engl J Med 321: $1754-1756,1989$

2. Xiping Z, Hua T, Hanqing C, et al: The protecting effects and mechanisms of Baicalin and Octreotide on heart injury in rats with SAP. Mediators Inflamm 2007: 19469, 2007.
3. Woo AY, Cheng $\mathrm{CH}$, and Waye MM: Baicalein protects rat cardiomyocytes from hypoxia/reoxygenation damage via a prooxidant mechanism. Cardiovasc Res 65: 244-253, 2005.

4. Burashnikov A, Petroski A, Hu D, et al: Atrial-selective inhibition of sodium channel current by Wenxin Keli is effective in suppressing atrial fibrillation. Heart Rhythm 9: 125-131, 2012.

5. Hu X, Wu B, Wang X, et al: Minocycline attenuates ischemia-induced ventricular arrhythmias in rats. Eur J Pharmacol 654: 274-279, 2011.

6. Demiryurek AT, Yildiz G, Esiyok S and Altug S: Protective effects of poly (ADP-ribose) synthase inhibitors on digoxin-induced cardiotoxicity in guinea-pig isolated hearts. Pharmacol Res 45: 189-194, 2002.

7. Imani A, Faghihi M, Sadr SS, et al: Noradrenaline reduces ischemia-induced arrhythmia in anesthetized rats: involvement of alpha1-adrenoceptors and mitochondrial K ATP channels. J Cardiovasc Electrophysiol 19: 309-315, 2008.

8. Nishimura N, Reien Y, Matsumoto A, et al: Effects of nicorandil on the cAMP-dependent Cl-current in guinea-pig ventricular cells. J Pharmacol Sci 112: 415-423, 2010.

9. Tsuburaya R, Yasuda S, Ito Y, et al: Shimokawa $\mathrm{H}$, Eicosapentaenoic acid reduces ischemic ventricular fibrillation via altering monophasic action potential in pigs. $\mathrm{J}$ Mol Cell Cardiol 51: 329-336, 2011.

10. Ravingerova T, Matejikova J, Pancza D and Kolar F: Reduced susceptibility to ischemia-induced arrhythmias in the preconditioned rat heart is independent of PI3-kinase/Akt. Physiol Res 58: 443-447, 2009.

11. Rosen MR and Hoffman BF: Mechanisms of action of antiarrhythmic drugs, Circ Res 32: 1-8, 1973.

12. Gettes LS: The electrophysiologic effects of antiarrhythmic drugs. Am J Cardiol 28: 526-535, 1971.

13. Wang M, Yu YB and Huang SE: Clinical observation on effect and safety of combined use of wenxin granule and amiodarone for conversion of auricular fibrillation. Zhongguo Zhong Xi Yi Jie He Za Zhi 26: 445-448, 2006 (In Chinese).

14. Xie PY and Shen SH: Effect of combination of Chinese and Western medicines on sinus rhythm maintenance in patients with auricular fibrillation after conversion. Zhongguo Zhong Xi Yi Jie He Za Zhi 26: 644-646, 2006 (In Chinese).

15. Zhou F, Hu SJ and Mu Y: Protection effect of Wenxin Keli on isoproterenol induced heart failure in rats. Zhongguo Zhong Yao Za Zhi 32: 1676-1679, 2007 (In Chinese).

16. Kalifa J and Avula UM: The Chinese herb extract Wenxin Keli: atrial selectivity from the Far East. Heart Rhythm 9: 132-133, 2012.

17. January CT, Riddle JM and Salata JJ: A model for early afterdepolarizations: induction with the $\mathrm{Ca}^{2+}$ channel agonist Bay K 8644. Circ Res 62: 563-571, 1988.

18. January CT and Riddle JM: Early afterdepolarizations: mechanism of induction and block. A role for L-type $\mathrm{Ca}^{2+}$ current. Circ Res 64: 977-990, 1989.

19. Marban E, Robinson SW and Wier WG: Mechanisms of arrhythmogenic delayed and early afterdepolarizations in ferret ventricular muscle. J Clin Invest 78: 1185-1192, 1986.

20. De Ferrari GM, Viola MC, D'Amato E, et al: Distinct patterns of calcium transients during early and delayed afterdepolarizations induced by isoproterenol in ventricular myocytes. Circulation 91: 2510-2515, 1995

21. Liu QN, Zhang L, Gong PL, et al: Daurisoline suppressed early afterdepolarizations and inhibited L-type calcium current. Am J Chin Med 38: 37-49, 2010.

22. Zobel C, Rana OR, Saygili E, et al: Mechanisms of $\mathrm{Ca}^{2+}$-dependent calcineurin activation in mechanical stretch-induced hypertrophy. Cardiology 10: 7281-7290, 2007.

23. Sucharov CC, Mariner PD, Nunley KR, et al: A beta1-adrenergic receptor CaM kinase II-dependent pathway mediates cardiac myocyte fetal gene induction. Am J Physiol Heart Circ Physiol 291: H1299-H1308, 2006.

24. Cano-Abad MF, Villarroya M, Garcia AG, et al: Calcium entry through L-type calcium channels causes mitochondrial disruption and chromaffin cell death. J Biol Chem 276: 39695-39704, 2001.

25. Guo W, Xu H, London B and Nerbonne JM: Molecular basis of transient outward $\mathrm{K}^{+}$current diversity in mouse ventricular myocytes. J Physiol 521: 3587-3599, 1999. 